\title{
Concepción metodológica con enfoque multidisciplinario para la exclusión e inclusión educativa en la formación profesional
}

Methodological conception with a multidisciplinary approach for educational exclusion and inclusion in vocational training.

\begin{abstract}
Concepção metodológica com abordagem multidisciplinar para a exclusão educacional e inclusão no ensino e treinamento vocacional
\end{abstract}

Osvaldo Pedro Santana-Borrego
https://orcid.org/0000-0002-3426-3138
Cuba
Universidad de Ciencias Médicas de La Habana
Geraldo Luis-Valdez
https://orcid.org/0000-0002-4470-5200
Cuba
Universidad de Ciencias Médicas de La Habana
Roxana Milagros Oviedo-Salazar
https://orcid.org/0000-0002-3908-1579
Facultad de Tecnología de la Salud
Cuniversidad de Ciencias Médicas de La Habana
Cuba
Recibido: 7/4/2021 • Aceptado: 20/5/2021

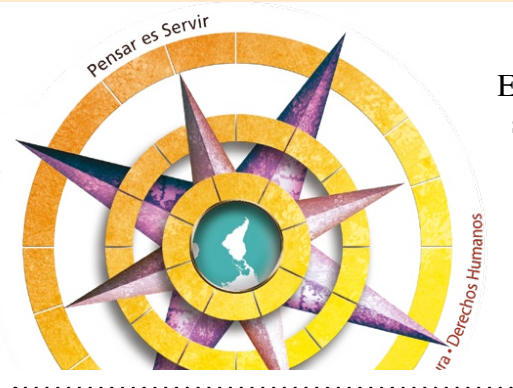

Resumen

El estudio sobre la exclusión e inclusión socioeducativa es una necesidad inaplazable para el mundo académico contemporáneo como proceso que demanda por su impronta social, la concepción metodológica 
con enfoque multidisciplinario para la formación profesional en la enseñanza superior cubana. El artículo recoge algunas hipótesis sobre el perfeccionamiento de la NUC ante los desafíos que enfrenta por los condicionamientos socioculturales que acontecen en el país para la construcción de una sociedad próspera y sostenible. El tópico intenta poner al relieve disímiles valoraciones sobre la creación, la innovación y la transferencia de conocimientos científicos, que permitan enfrentar el cambio socioeducativo e impulsar el progreso científico del país inmerso en un escenario cada vez más convulso engendrado por la globalización neoliberal y el conjunto de acciones que se articulan en la conceptualización del Modelo Económico y Social de desarrollo socialista cubano.

Palabras claves: concepción metodológica, enfoque exclusión e inclusión socioeducativa, Cuba, modelo educativo

\begin{abstract}
The study on socio-educational exclusion and inclusion is an urgent issue for the contemporary academic world as a process that demands, due to its social imprint, of a methodological conception with a multidisciplinary approach for professional training in Cuban higher education. The article gathers some hypotheses on the improvement of the NUC in view of the challenges it faces due to the socio-cultural conditions that occur in the country for the construction of a prosperous and sustainable society. In this sense, the topic tries to highlight dissimilar assessments on the creation, innovation and transfer of scientific knowledge, which allow facing the socio-educational change and promoting the scientific progress of the country immersed in an increasingly convulsive scenario generated by neoliberal globalization and the set of actions that are articulated in the Conceptualization of the Economic and Social Model of Cuban socialist development.
\end{abstract}

Keywords: Methodological conception, Focus on exclusion and socioeducational inclusion, Cuba, educational model

Resumo

O estudo sobre exclusão e inclusão sócio-educativa é uma necessidade urgente para o mundo acadêmico contemporâneo como um processo que exige sua marca social, a Concepção Metodológica com uma abordagem multidisciplinar para a formação profissional no ensino superior cubano. $\mathrm{O}$ artigo reúne algumas hipóteses sobre a melhoria da NUC diante dos desafios que enfrenta devido às condições socioculturais que ocorrem no país para a construção de uma sociedade próspera e sustentável. Neste sentido, o tema tenta destacar valorizações diferentes sobre a criação, inovação e transferência do conhecimento científico, que permitem enfrentar a mudança 
sócio-educativa e promover o progresso científico do país imerso em um cenário cada vez mais convulsivo gerado pela globalização neoliberal e pelo conjunto de ações que se articulam na Conceptualização do Modelo Econômico e Social do desenvolvimento socialista cubano.

Palavras chave: Concepción metodológica, Enfoque de exclusión e inclusión socioeducativa, Cuba, modelo educativo

\section{Introducción}

Las actuales dinámicas que generan las políticas socioeducativas en la enseñanza superior cubana como parte de la universalización de la universidad, están en estrecha e intrínseca relación con la Agenda 2030 de desarrollo sostenible (ONU, 2015). Perspectiva en la cual las universidades articulan acciones de perfeccionamiento del conocimiento científico hacia una instrucción y formación inclusiva de calidad de sus egresados para la transformación social, y, por otra parte, enfocada hacia los problemas primordiales de la educación, la sociedad y la investigación desde un paradigma científico-teórico en concordancia con su momento histórico social.

En este contexto tan complejo como dinámico, se reproducen sistemas de relaciones que generan cambios en las estructuras de funcionamiento de la universidad, en el cual no ha sido ajena la articulación de profundas transformaciones sociales y educacionales que confluyen y se interconectan procesos de exclusión e inclusión socioeducativa que meritan investigar por su impronta en sus resultados. Por ello se requiere continuamente de un abordaje a partir de reflexiones y enfoques de la academia como institución docente de carácter profesional en el marco de la conceptualización de la Nueva Universidad Cubana (NUC) (Colectivo de autores, 2012).

Es una concepción que pretende relevar la pertinencia social en el campo de la investigación educativa y la necesidad inaplazable de su análisis para el mundo académico contemporáneo. En este tejido de relaciones, la institución docente demanda como encargo social en el siglo XXI, múltiples miradas en su quehacer científico en víspera de analizar los desafíos que debe asumir la NUC como responsabilidad y cambio social en la Cuba de hoy, inmersa en un escenario cada 
vez más convulso engendrado por factores como la globalización (neoliberal) y el perfeccionamiento de la educación superior cubana.

En este sentido, el tema de la exclusión e inclusión socioeducativa amerita de su indagación en los sistemas formativos, y, por otra parte, exige proponer desde un enfoque multidisciplinario, la mirada oportuna de los profesionales universitarios como perspectiva de desarrollo, hacia la construcción de otras maneras de hacer, si aspiramos a que la educación, realmente, contribuya al desarrollo de sociedades más justas, democráticas y solidarias.

Sobre esta mirada, el artículo aspira a mostrar prácticas educativas y de culturas inclusivas interconectadas con la realidad social. Asimismo, la necesidad de contextualizar métodos de enseñanza y aprendizaje que respondan al espacio-tiempo para impulsar el perfeccionamiento de la educación con iniciativas y teorías inclusivas de calidad en su contexto (MES, 2018).

El proceso de exclusión e inclusión socioeducativa, constituye en sí mismo, un asunto de esmerada atención, debido que se encuentra al margen de la vida social, que tiende a generalizarse de manera invisible, aun cuando se convierte en un fenómeno de la realidad social dentro de un contexto histórico cultural globalizado en el que nos vemos inmersos. De este modo se podrían analizar los significados e implicaciones que se esconden tras algunas prácticas educativas calificadas como inclusivas que, sin embargo, no hacen más que perpetuar el estatus del sistema y abrir nuevas puertas a la marginación... (Parrilla, 2007).

En el artículo se presenta un conjunto de implicaciones que favorecen o limitan el desarrollo de las estructuras de los procesos docentes dentro del perfeccionamiento formativo y a su vez, aportan distintas perspectivas al conocimiento y la discusión en torno a este fenómeno educativo. Asimismo, muestra la contradicción entre la necesidad de articular procedimiento innovadores de inclusión que favorezcan la concepción de enseñanza-aprendizaje en la formación del profesional docente y la propuesta de diseñar una metodológica con enfoque multidisciplinario para la exclusión e inclusión en la NUC que permite establecer como objetivo general.

\footnotetext{
4 Concepción metodológica con enfoque multidisciplinario para la exclusión e inclusión educativa en la formación profesional Osvaldo Pedro Santana-Borrego, Geraldo Luis-Valdez y Roxana Milagros Oviedo-Salazar
} 
Por la importancia y novedad que se debate, el tema es en su esencia misma, un asunto que postula una lectura especial de los aportes científicos, el cual permite comprender y justificar el estudio. Sobre este particular, se cita oportunamente un grupo de valiosas contribuciones y reflexiones de diversas ramas de la ciencia, que por su amplio caudal de información se relaciona de forma sintetizada a continuación.

En este contexto, se consultaron los informes estadísticos que aporta la Oficina Regional de Educación de la UNESCO; la información que se ofrece en torno al tema de la Agenda de desarrollo 2030; la concepción del Modelo Económico y Social de Desarrollo Socialista y su reconocimiento jurídico en la Constitución Socialista aprobada en el año 2019; los resultados de investigaciones científicas del Congreso Internacional Pedagogía 2020 y 2021; el compendio de resultados científicos del Centro de Investigaciones Psicológicas y Sociológicas (CPIS), los resultados de Consejo Latinoamericano de Ciencias Sociales (CLACSO) y el Programa Nacional de Ciencia Tecnología e Innovación.

\section{Desarrollo}

Las mutaciones sociales, educacionales, tecnológicas, y sus influencias como metamorfosis en la formación profesional, transfiguran el escenario y producen una nueva cartografía del espacio social y educativo que demanda valorizar sus interacciones.

A partir de estos argumentos, resulta evidente proponer una concepción metodológica desde un enfoque multidisciplinario en la formación profesional. Estrategia de trabajo que impone como desafíos en la NUC, articular nuevas prácticas de la comunicación inclusiva, la activa participación docente como empoderamiento social en el escenario pedagógico y una cultura de la inclusión mediada por el conjunto de premisas, principios, objetivos, categorías y valores con que sus miembros se identifican, dee tal modo, que orienten sus acciones al intercambio de saberes, tecnologías y actúen sobre los procesos de la vida social y en las decisiones que lo determinan, como se muestra en el siguiente gráfico: 
Gráfico 1. Concepción metodológica con enfoque multidisciplinario para la exclusión e inclusión educativa en la formación profesional.

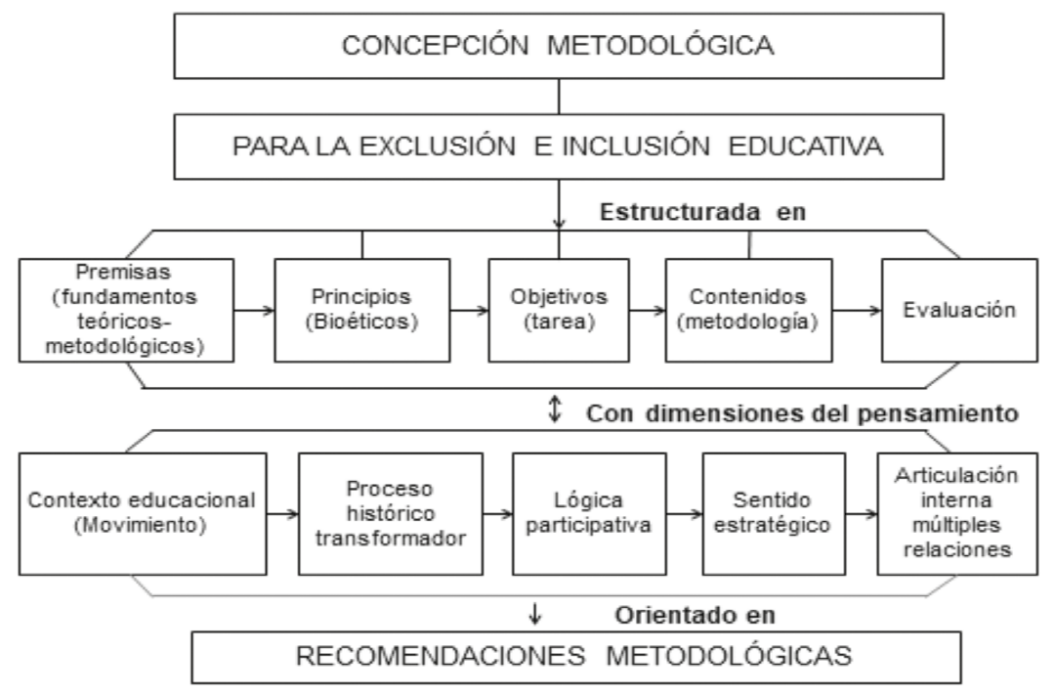

Fuente: Elaboración de los autores.

En este sentido, los resultados sobre la exclusión e inclusión educativa constituyen necesidades objetivas, $\mathrm{y}$, por consiguiente, generan buenas prácticas a consultar como herramientas de análisis desde enfoques multidisciplinarios. Este proceso articulado como hecho social, ha sido históricamente visto por académicos, instituciones científicas, tesis de doctorados y de maestrías como encargo de universidades en trabajos de cursos, los cuales aportan una amplia gama de teorías y evidencias que valorizan el objeto de estudio.
Según Echeita (2013) es un concepto teórico de la pedagogía y una práctica poliédrica. Surge como consecuencia de los altos niveles de exclusión y desigualdades educativas que persisten en la gran mayoría de los sistemas, a pesar de los significativos esfuerzos que han invertido para incrementar la calidad y la equidad de la educación, objetivo principal de las reformas de la región.

Por su impronta y necesidad de sus proyecciones de transformación socioeducativa, aún carecen de una

\footnotetext{
6 Concepción metodológica con enfoque multidisciplinario para la exclu-

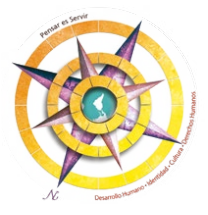


definición clara. Es un concepto en constante actualización que responde a la propia dialéctica de desarro1lo. En este sentido, existe escasa literatura que incide en la familiarización de la temática con el contexto educacional cubano. Aspecto que genera la posterior apertura de nuevas líneas de investigación científica que contribuyan a profundizar y complejizar el abordaje del fenómeno en cuestión por la NUC.

La propuesta de concepción puede constituir una herramienta teórico-metodológica que puede ser empleada como aparato referencial e instrumento de medición y control social. Asimismo, genere desde sus efectos, documentos normativos y disposiciones legales que regulen la práctica pedagógica contemporánea en torno a los procesos que hoy desarrolla la enseñanza superior como política de desarrollo, lo cual condicionó que la tipología de investigación utilizada en la indagación científica y en correspondencia con sus peculiaridades y los objetivos de los que se partió, indujeron la utilización de una perspectiva metodológica mixta, predominantemente, cualitativa de corte microsocial.

$\mathrm{Al}$ señalar las características del tema y la compleja realidad que motiva su estudio, se concibe un diseño metodológico de forma flexible que facilita desde la práctica investigativa, admitir la construcción de teorías. De tal modo, el tipo de investigación aplicada es de naturaleza descriptiva-explicativa, la cual tuvo como propósito, realizar un acercamiento a los procesos de exclusión e inclusión socioeducativa desde un enfoque multidisciplinario apoyada en la investigación-desarrollo y con la utilización de métodos y técnicas para obtener la información tales como: análisis de documentos, cuestionario, entrevista individual y grupal, dinámica grupal, los cuales se combinaron con el análisis de los discursos de actores sociales que conformaron la población en estudio y una exhaustiva revisión de textos y documentos que incluyeron o no, de cierta forma, elementos constitutivos de las categorías que se abordan y proporcionaron la siguiente interrogante.

\section{¿Por qué una concepción metodológica?}

La concepción metodológica parte de la construcción teórica que permita realizar propuestas metodológicas de instrumentación, y a su vez, describir, estructurar y representar integradoramente, nuevos procederes sociopedagógicos. (Ganelin, 2001). 
En concordancia con el autor, está dada porque el contenido objetivo y subjetivo del sistema de valores que varía metodológicamente en correspondencia con las tendencias del desarrollo social. La forma en que se concibe puede proporcionar tareas que, por su naturaleza inductora, direccionan hacia el desarrollo del pensamiento crítico e innovador identitario y científico que debe ser evaluada en la actividad práctica cognoscitiva.

Según la Dra. Oviedo, 2019, esta concepción demanda que la evaluación favorezca tareas que rompan los modelos transmisivos de información... que, por lo general, son aprendidos de manera acrítica... que contribuyan a nuevas revalorizaciones, para generar verdaderos espacios de discusión científica (Oviedo, 2019). Posicionamiento que resulta de gran interés, ya que se incorporan aportes desde el enfoque pedagógico e histórico cultural para la interpretación de los procesos de exclusión e inclusión socioeducativo en la formación profesional.

Su carácter novedoso constituye una invitación al cambio de mentalidad, a una visión creativa e innovadora con relación al proceso de exclusión e inclusión socioeducativa, de la formación y la actualización del rol profesional, de la aplicación de nuevos conceptos, estrategias y acciones que demuestren en la práctica como actividad humana y criterio valorativo del conocimiento, la contribución dentro de un nuevo modelo de universidad, a la formación integral del ser humano para la transformación y del sujeto mismo como proceso social.

De acuerdo con este concepto, los centros de educación superior no solo deben promover la formación de especialistas de alta calificación, sino también producir investigaciones encaminadas a solucionar los principales problemas de la ciencia en general, y a emplear estos conocimientos para satisfacer las necesidades más perentorias del país.

Coherentemente, en Cuba las transformaciones que se vienen produciendo en la educación superior, como parte de la estrategia de su perfeccionamiento, han derivado, entre otros, en el diseño de una nueva generación de planes de estudio. Este resultado ha obligado a reflexionar sobre la necesidad de revisar y proponer cambios en las regulaciones vigentes que aseguren los niveles de calidad deseados en el proceso de formación integral de los futuros profesionales del país (MES, 2018). 
En el caso particular de Cuba la educación está sometida a cambios medulares a partir de las transformaciones derivadas de investigaciones cuyas bases están en los diversos criterios aportados por Serra (2002), Borges (2012), MINED (2015), Pérez Galdó y Hernández Pérez (2015); todos aportan un elemento en común: el derecho de toda persona a participar en la sociedad.

Ello implica la construcción de iniciativas inclusivas, nuevas concepciones metodológicas, habilidades, formas de organización de la enseñanza, evaluación, medios didácticos, capacidades de decisión, y la democratización de las estrategias participativas, que incidan de manera significativa, en la reducción de la exclusión educativa ante los nuevos retos que comprometen el pensamiento universitario del siglo XXI como proyección social.

¿Qué insuficiencias sustentan una concepción metodológica con enfoque multidisciplinario, para la exclusión e inclusión educativa en la formación docente como situaciones problemáticas? La investigación aporta desde el propio diagnóstico, la observación participante y el conocimiento empírico, un conjunto de insuficiencias que actúan en los sistemas educacionales que, por el propio desarrollo universitario, pueden conducir al debate desde la oportuna visión crítica a la realidad, algunas de las cuales se mencionan a continuación.

\section{Insuficiente:}

- Desaprovechamiento multidisciplinarios para articular estrategias participativas que faciliten procesos de inclusión.

- Estrategias didácticas y metodologías inclusivas.

- Sistemas informativos-comunicativos-participativos como herramienta de trabajo docente.

- Visibilidad en la identificación de acciones exclusivas.

- Concepción de los procesos de inclusión educativa desde el paradigma universitario para la formación profesional.

En consecuencia, con el progreso de la universidad, ciencia, tecnología y desarrollo sostenible que acontece en la NUC, el Dr. Núñez Jover en el año 2017, plantea que es conveniente avanzar hacia un modelo de educación superior que promueva este último aspecto, incluida la lucha contra la pobreza y la inclusión social. Ese debate está teniendo lugar 
en América Latina y es promovido por la Unión de Universidades de América Latina (Núñez, 2017).

Desde los enfoques educacionales en la NUC, se demuestra la necesidad de proponer una concepción metodológica que dé salida a un conjunto de limitaciones que se vislumbran en la realidad socioeducativa contemporánea, desde el paradigma universitario que reclaman de un enfoque multidisciplinario. Estas reflexiones podrían representar una oportunidad para precisar el estatus de la distinción entre inclusión y exclusión dentro de la teoría sociológica. En relación con ella, hay que partir de que no se trata de si los individuos son parte de la sociedad o no. La distinción inclusión-exclusión es una interna al sistema, lo que significa, en nuestro caso, que solo debe ser aplicada en el orden de la comunicación y la participación inclusiva como empoderamiento para la transformación de realidades.

En ese orden, la investigación analiza la exclusión como un efecto secundario que al igual que toda fijación de una identidad desconside$\mathrm{ra}$, es algo que no les pertenece a las estructuras educacionales. La cara interna de esta forma, la inclusión, disfruta de atención preferente como necesidad para el cambio social.
Es en consecuencia, una realidad que hace necesario un esfuerzo especial para, dirigir la observación hacia la exclusión como fenómeno dentro de los cambios que se conciben desde la universalización de la enseñanza superior, para promover la conceptualización que permita la apropiación de espacios investigativos

En esta línea de pensamiento, Echeita, en su artículo "Inclusión y exclusión educativa. De nuevo "voz y quebranto" (2008), esboza que la inclusión es hoy una aspiración de todos los sistemas educativos de Iberoamérica. No podría ser de otra manera si aspiramos a que ella, realmente, contribuya al desarrollo de sociedades más justas, democráticas y solidarias.

La preocupación por la inclusión en Iberoamérica, surge como consecuencia de los altos niveles de exclusión y de las desigualdades educativas que persisten en la gran mayoría de los sistemas a pesar de los significativos esfuerzos que han invertido para incrementar la calidad y la equidad, objetivo principal de las reformas educacionales de la región.

En esta lógica de pensamiento, resulta evidente resaltar, que, si bien la educación no es la única llave para cambiar el estado de situación,

10 Concepción metodológica con enfoque multidisciplinario para la exclu-

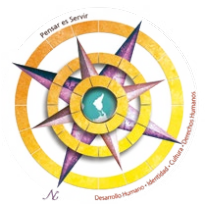


debiera disponer de todos los medios a su alcance, diseñar nuevas acciones participativas, comunicacionales y culturales inclusivas para evitar que las desigualdades aumenten o se profundicen, como consecuencia de las deficiencias y las limitaciones de la propia actuación educativa.

En tal sentido, los sistemas educativos debieran doblar los esfuerzos para equiparar las oportunidades de los disímiles actores que forman y son parte de las políticas educacionales y generar mejores condiciones de partida, que participen desde la ética de actuación, y permitan compensar sus diferencias de entrada.

\section{Conclusiones}

Los desafíos que impone la NUC demanda como propuesta de cambio, una concepción metodológica que involucre a los actores socioeducativos inmersos en los sistemas educacionales. De tal forma, se convierte en sí misma en una herramienta teórico y práctica que, desde el pensamiento científico, puede ser asumida con corresponsabilidad y compromiso social.

En tal sentido, debe estar sustentada en criterios y principios metodológicos de carácter científico, que permita estructurar toda una estrategia de trabajo que consiga planificar, disenar, ejecutar, evaluar y sistematizar procesos ordenados y coherentes en la NUC.

La propuesta demanda, a su vez, una secuencia lógica e innovadora que aporte por sus resultados, a la transformación cualitativa de la situación de la cual se partió.

\section{Referencias bibliográficas}

Asamblea General de la ONU. (2015). Agenda 2030 de desarrollo sostenible. Disponible en: https://www. google.com.

AAVV. (2012). Preparación pedagógica para profesores de la Nueva Universidad Cubana. La Habana: Editorial Universitaria Félix Varela. Disponible en: eduniv.reduniv.edu.cu

Echeita, G. Inclusión y exclusión educativa: de nuevo "Voz y Quebranto". REICE, Revista Iberoamericana sobre Calidad, Eficacia y Cambio en Educación, 11(2), 99-118. Disponible en: https://scholar.google.com.cu

Ganelin, I. (2001). La asimilación consciente en la escuela. Colección Pedagógica. Editorial. Grijalbo, S. A. México.

Oviedo, R. (2019). Concepción metodológica para los estudios de la medicina natural en Tecnología de la Salud mediante la utilización del pensamiento crítico. Tesis de Doctorado en Ciencias de la Educación Médica. 
Parrilla, A. (2007). Inclusive Education in Spain: a view from inside. En L. Barton y F. Amstrong (Eds.) Policy, Experience and Change: Cross Cultural Reflections on Inclusive Education.

Ministerio de Educación Superior (MES), República de Cuba. Resolución $n$. 2/18. Disponible en: www.gacetaoficial.gob.cu

Núñez, J; García, R. (2017). Universidad, ciencia, tecnología y desarrollo sostenible. En Revista Espacio, Vol. 38, (N. $\left.{ }^{\circ} 39\right)$. Disponible en: www.revistaespacios.com. 